\title{
Experimental and theoretical study of the radial density distributions of large ${ }^{3} \mathrm{He}$ droplets
}

\author{
Jan Harms and J. Peter Toennies* \\ Max-Planck-Institut für Strömungsforschung, D-37073 Göttingen, Germany \\ Manuel Barranco and Marti Pi \\ Departament d'Estructura i Constituents de la Matèria, Facultat de Fisica, Universitat de Barcelona, E-08028 Barcelona, Spain
}

(Received 19 September 2000; published 20 April 2001)

\begin{abstract}
The integral cross sections of large ${ }^{3} \mathrm{He}$ droplets and the number of atoms in the corresponding droplets $\left(\bar{N}=2 \times 10^{3}-2 \times 10^{4}\right.$ atoms $)$ have been measured in molecular-beam scattering experiments. The experimental results are in very good agreement with integral cross sections calculated from the radial density distributions predicted from density-functional theory calculations. The experimentally confirmed theoretical $10-90 \%$ surface thicknesses vary between $8.0 \AA\left(\bar{N}=10^{3}\right)$ and $7.6 \AA\left(\bar{N}=4 \times 10^{4}\right)$ and are about $30 \%$ larger than calculated for ${ }^{4} \mathrm{He}$ droplets of similar sizes.
\end{abstract}

DOI: 10.1103/PhysRevB.63.184513

\section{INTRODUCTION}

There is great current interest in helium clusters and droplets. ${ }^{1-3}$ Being the only definitely liquid clusters they provide a uniquely gentle homogeneous and at the same time very cold matrix for high-resolution spectroscopy of single molecules or aggregates of molecules. ${ }^{4-6}$ Recent experiments have used the highly resolved spectra of single embedded molecules to probe the physical properties of the droplets. ${ }^{6-8}$ Experimental studies ${ }^{9}$ of the phonon wings of vibronic transitions have shown that ${ }^{4} \mathrm{He}$ droplets are superfluid, in agreement with theoretical predictions. ${ }^{10,11}$ In the infrared spectral region unexpected sharp rotational lines have been observed for several embedded molecules, such as $\mathrm{SF}_{6}$ (Refs. 6 and 7) and $\mathrm{OCS},{ }^{8}$ whereas in ${ }^{3} \mathrm{He}$ droplets, which are not expected to be superfluid, the same molecules show a collapsed spectrum with only one relatively broad peak. ${ }^{8}$ These observations have been interpreted as indicating that the sharp rotational structure is due to the superfluidity of the ${ }^{4} \mathrm{He}$ droplets. This new microscopic manifestation of superfluidity has been called "molecular superfluidity.' The same infrared experiments also provide information on the droplet temperatures which are $0.38 \mathrm{~K}$ for ${ }^{4} \mathrm{He}$ and $0.15 \mathrm{~K}$ for ${ }^{3} \mathrm{He}$ droplets. ${ }^{7}$ Recently it has been demonstrated that by using ${ }^{4} \mathrm{He} /{ }^{3} \mathrm{He}$ mixed droplets the lower temperatures provided by the evaporation from the outer layers of ${ }^{3} \mathrm{He}$ can be combined with the superfluid environment of the inner layers of ${ }^{4} \mathrm{He}$ next to the probe molecule to obtain even sharper spectral features. ${ }^{12}$

Droplets consisting of pure ${ }^{3} \mathrm{He}$ atoms are also of great fundamental interest since ${ }^{3} \mathrm{He}$ is the only neutral Fermi liquid accessible to experiments. As a finite-sized Fermi system with a simple well-known interatomic potential ${ }^{3} \mathrm{He}$ droplets are ideal model systems for understanding the effects of Fermi statistics in nuclei and neutron stars where the interactions are much more complicated. As in the case of nuclei the ambient droplet temperatures are well below the estimated bulk Fermi temperature $T_{F}=\hbar^{2} k_{F}^{2} / 2 m^{*} \approx 1.75 \mathrm{~K}$, for $k_{F}=0.79 \AA^{-1}$ and $m^{*}=2.80 m_{3}$, where $m_{3}$ is the mass of a free ${ }^{3} \mathrm{He}$ atom. ${ }^{13}$

Whereas many properties of ${ }^{4} \mathrm{He}$ droplets have been stud-
PACS number(s): 67.55.-s, 61.46.+w, 36.40.Mr

ied theoretically and experimentally, ${ }^{1}$ far less is presently known about ${ }^{3} \mathrm{He}$ droplets. The mass spectra of ${ }^{3} \mathrm{He}$ droplets produced in cryogenic free jet expansions were measured many years ago. ${ }^{14,15}$ More information is available from extensive theoretical studies of the structure and energetics of ${ }^{3}$ He droplets. ${ }^{16-30}$ The most recent calculations predict that whereas ${ }^{4} \mathrm{He}$ clusters from the dimer upwards are all stable, ${ }^{3}$ He clusters with less than 29 atoms are all unstable. ${ }^{26}$ This is attributed to the large zero-point energy of the ${ }^{3} \mathrm{He}$ atoms resulting from their smaller mass and because of their Fermion nature. Previously the density distributions of ${ }^{3} \mathrm{He}$ clusters with several tens of atoms up to droplets with $N$ $=1360$ atoms $^{23}$ have been calculated using a variety of different approximations. ${ }^{16-18,23,27}$ Stringari has estimated the thickness of the surface of small clusters with $N$ up to 168 atoms by assuming a generalized Fermi function,

$$
\rho(R)=\frac{\rho_{0}}{\left[1+\exp \left(\frac{R-R_{0}}{a}\right)\right]^{\nu}},
$$

where $a$ is a width parameter and $R_{o}$ is the radius for a liquid droplet with a constant density and sharp edge. For this profile function the 10-90\% surface thickness $t$ is given by

$$
t=a \cdot \ln \left(\frac{10^{1 / \nu}-1}{\left(\frac{10}{9}\right)^{1 / \nu}-1}\right),
$$

which reduces to $t=4 a \ln 3$ for symmetric $(\nu=1)$ density profiles for which Stringari estimated $t \approx 7 \AA .{ }^{16}$ This result can be compared with the thickness of the surface of bulk liquid ${ }^{3} \mathrm{He} t=8.3 \AA$ obtained using a local energy density functional (DF). ${ }^{29}$

More recently Barranco et al. have calculated the bulk liquid-free surface density profiles at finite temperatures and found that at temperatures below $1 \mathrm{~K}$ the density profiles were nearly independent of the temperature. ${ }^{30}$ At present we are not aware of any experimental determinations of the liquid surface density profile with which to compare these results.

The present study parallels in many respects an earlier combined experimental and theoretical scattering investiga- 
tion of the radial density profiles in ${ }^{4} \mathrm{He}$ droplets, ${ }^{31}$ which will be referred to as I throughout the present paper. The basic idea of the experiment is to measure the effective droplet integral cross sections by scattering the droplets from a beam of krypton atoms. From the cross section the cluster volume is determined. The angular distribution of the deflected droplets, measured in the same experiment, depends on the mean number of atoms and the number distribution of the same droplets. From these two measured quantities the average density within the liquid drop model can be ascertained. As found for the ${ }^{4} \mathrm{He}$ droplets the experimental average density of the ${ }^{3} \mathrm{He}$ droplets is significantly less in this case, by up to $30 \%$, than that of bulk helium. Since all theoretical calculations indicate that the central density of even small helium clusters with about 300 or more atoms is roughly equal to the bulk density the reduced value of the average density is attributed to deviations from the liquid drop model coming from the falloff in the density in the outer region. As in the previous work, the measured effective integral cross sections versus mean atom number size is compared with the values extracted from density distributions based on density-functional theory. The very good agreement found provides further confirmation of the validity of the experimental procedures as well as of the density functional used. Moreover, the good agreement indirectly supports the assumption that the droplets are rather spherical as discussed in the earlier work.

For the ${ }^{3} \mathrm{He}$ droplets the calculated surface density profile is found to be asymmetric. The $10-90 \%$ thickness varies between $t=8.0 \AA \quad\left(\bar{N}=10^{3}\right)$ and $t=7.6 \AA \quad\left(\bar{N}=4 \times 10^{4}\right)$, somewhat smaller than the $t=8.3-\AA$ thickness of the bulk liquid surface. ${ }^{29}$ If the density decrease in the outer region is assumed symmetric with respect to the 50\% dropoff radius the thickness extracted directly from the experimental data is smaller than the density functional calculations by about $\Delta t$ $=2 \AA$. This observation and the good fit of the data with an asymmetric profile provide experimental evidence that the density falloff is not symmetric.

The apparatus and measurement procedures are described briefly in Sec. II A. The experimental results are presented in Sec. II B. In Sec. III the density-functional calculations are described and compared with experiments. The paper closes with a brief summary.

\section{EXPERIMENT AND MEASUREMENT PROCEDURES}

\section{A. Apparatus}

The molecular-beam scattering apparatus has been described in some detail in several recent articles. ${ }^{32-34}$ Essentially the ${ }^{3} \mathrm{He}$ droplets are produced by a free jet expansion from a stagnation source chamber at a high pressure $\left(P_{0}\right.$ $=10-40$ bars $)$ and a low temperature $\left(T_{0}=7-14 \mathrm{~K}\right)$ through a thin walled nozzle with $5 \pm 1 \mu \mathrm{m}$ diameter. The ${ }^{3} \mathrm{He}$ gas used is $99.9 \%$ pure with the remaining $0.1 \%$ impurity being mostly ${ }^{4} \mathrm{He}$. This small amount of ${ }^{4} \mathrm{He}$ is concentrated at the center of the droplet ${ }^{35}$ and cannot affect the surface properties of the system. The droplet velocity distributions were measured and found to be reasonably narrow
$(\Delta v / v \leqslant 2 \%)$ with mean speeds of between 250 and $400 \mathrm{~m} / \mathrm{s}$. After passing through a skimmer and several differential pumping stages the droplets are deflected by collisions with krypton atoms in a secondary beam which crosses the droplet beam at an angle of $40^{\circ}$. The $\mathrm{Kr}$ atom beam was produced in a free jet expansion $\left(P_{0} \approx 200 \mathrm{mbar}\right.$ and $\left.T_{0}=300 \mathrm{~K}\right)$ through a 40- $\mu \mathrm{m}$ diameter nozzle. These source conditions were chosen to avoid an appreciable amount of dimers or larger clusters, while still having a reasonably narrow velocity distribution $(\Delta v / v \approx 20 \%)$. A small portion (5-10\%) of the droplets is deflected by the momentum transfer imparted by the secondary beam gas atoms, most of which are captured by the droplets. ${ }^{33}$ After scattering the droplets are detected $885 \mathrm{~mm}$ downstream from the scattering center with an electron impact ionizer followed by a magnetic mass spectrometer set at the ${ }^{3} \mathrm{He}_{2}^{+}$mass of 6 atomic mass units (amu) which is a predominant decay fragment of larger cluster ions. The incident droplet beam is collimated to an angular half-width of about $1.2 \mathrm{mrad}$ and the angular resolution of the detector was $270 \mu \mathrm{rad}$. By rotating the detector around the scattering region in the plane of the two beams in small angular steps of only about $0.2 \mathrm{mrad}$ the angular distribution of the deflected droplets was measured. This provides information on the number sizes and size distributions of the droplets. With the detector set along the beam axis the attenuation of the incident droplet beam was measured with a detector angular resolution of about $1 \mathrm{mrad}$ and with the measured effective scattering density of the secondary beam (see Sec. II C) the size of the classical integral cross section can be ascertained. From these two measurements the average densities of the droplets are established.

\section{B. Number of atoms in the droplets}

Figure 1 shows a mass spectrum measured for a droplet beam containing about $3 \times 10^{3}$ atoms after capture of $\mathrm{Kr}$. The mass spectrum is dominated by a series of peaks spaced 3 amu apart which are due to the $\left({ }^{3} \mathrm{He}\right)_{n}^{+}$ion fragments. A distinct peak is found at $84 \mathrm{amu}$ which is due to $\mathrm{Kr}$ atoms which have been trapped in the droplet.

Figure 2 shows log plots of the scattered intensity [on the $\left({ }^{3} \mathrm{He}\right)_{2}^{+}$mass] as a function of the scattering angle for droplets of five different sizes. The upper curve is measured with the secondary beam crossing the droplet beam, whereas the lower curve is measured with the secondary beam intersected by a flag. The small difference between the deflected signal and the undeflected signal reduced by the attenuation factor, shown on a ten times larger scale below the angular distribution, is attributed to droplets which were deflected after capturing a secondary beam atom. Since from earlier experiments with ${ }^{4} \mathrm{He}$ droplets the momentum transfer is known to be complete the angle of deflection $\vartheta$ is directly dependent on the number $N$ of atoms in the droplet and is given by

$$
\begin{aligned}
\vartheta \approx \tan (\vartheta) & =\frac{m_{\mathrm{sec}} v_{\mathrm{sec}} \sin \alpha}{m_{\mathrm{drop}} N v_{\mathrm{drop}}+m_{\mathrm{sec}} v_{\mathrm{sec}} \cos \alpha} \\
& \approx \frac{m_{\mathrm{sec}} v_{\mathrm{sec}} \sin \alpha}{m_{\mathrm{drop}} N v_{\mathrm{drop}}},
\end{aligned}
$$




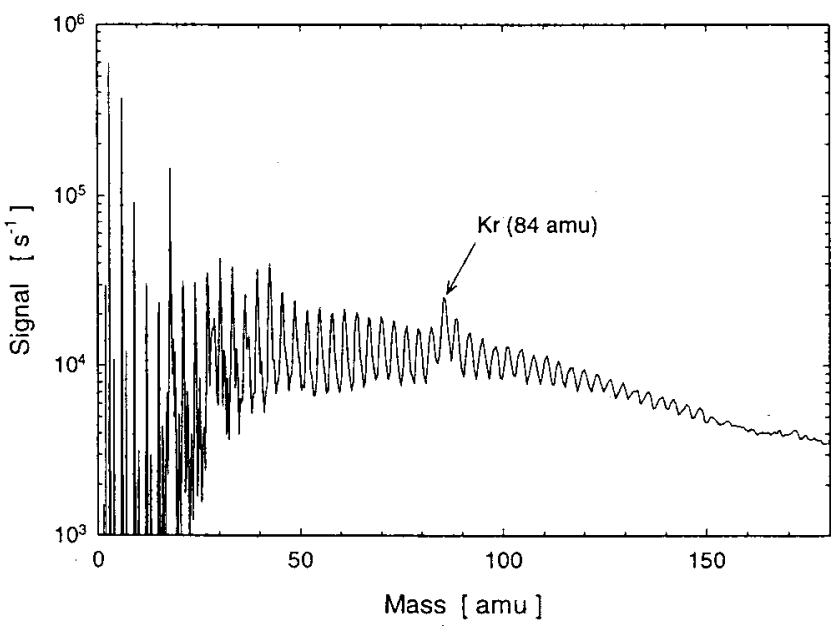

FIG. 1. A typical mass spectrum of a ${ }^{3} \mathrm{He}$ droplet beam $\left(P_{0}\right.$ $=25$ bars, $\left.T_{0}=9.5 \mathrm{~K}\right)$ with a mean number size $\bar{N}=3100$ measured after the pickup of $\mathrm{Kr}$ atoms. The atoms were captured in the scattering chamber from the gas emitted by the secondary beam but with the $\mathrm{Kr}$ beam flag closed. The scattering probability was about $25 \%$. The peak at $84 \mathrm{amu}$ is due to $\mathrm{Kr}$ atoms embedded inside the droplet. The other major peaks spaced 3 amu apart are ion fragments of the ${ }^{3} \mathrm{He}$ droplets. The large peak at $18 \mathrm{amu}$ is probably due to a small amount of captured water molecules.

where $m_{\text {drop }} N v_{\text {drop }}$ is the momentum of the droplets, $m_{\text {sec }} v_{\text {sec }}$ is the momentum of the secondary beam gas atoms, and $\alpha$ is the angle between the two beams.

The droplet size distributions are plotted on the right-hand side of Fig. 2 next to the corresponding measurements of the angular distributions. As found for ${ }^{4} \mathrm{He}$ droplets the measured droplet atom number distributions can be very well fitted with a log-normal distribution: ${ }^{31,32}$

$$
P(N)=\frac{1}{\sqrt{2 \pi} N \delta} \exp \left[-\frac{(\ln N-\mu)^{2}}{2 \delta^{2}}\right]
$$

where the mean number of atoms $\bar{N}$ and the width [full width at half maximum (FWHM)] of the distribution $\Delta N_{1 / 2}$ are

$$
\bar{N}=\exp \left(\mu+\frac{\delta^{2}}{2}\right)
$$

and

$$
\Delta N_{1 / 2}=\exp \left(\mu-\delta^{2}+\delta \sqrt{2 \ln 2}\right)-\exp \left(\mu-\delta^{2}-\delta \sqrt{2 \ln 2}\right)
$$

respectively. Table I lists the results for $\bar{N}, \Delta N_{1 / 2}, \mu$, and $\delta$, measured using $\mathrm{Kr}$ as the deflection atom for a wide range of different ${ }^{3} \mathrm{He}$ source pressures and temperatures. There it should be noted that the $\Delta N_{1 / 2}$ is comparable with $\bar{N}$. The mean sizes of ${ }^{3} \mathrm{He}$ droplets have also been recently measured as a function of the source stagnation pressures and temperatures using a technique based on an analysis of the relative depletion in the mass spectrometer detector signal after reso- nant photon absorption of an embedded molecule. ${ }^{12}$ These independent measurements agree nicely with the results listed in Table I.

Compared to ${ }^{4} \mathrm{He}$ droplets the mean ${ }^{3} \mathrm{He}$ droplet sizes reveal quite a different behavior, with changing source conditions. Whereas small ${ }^{4} \mathrm{He}$ clusters and droplets are obtained for a wide range of source pressures at source temperatures below about $T_{0}=30 \mathrm{~K},{ }^{3} \mathrm{He}$ droplets appear very suddenly as the source temperature for a given stagnation pressure approaches an isentrope with an entropy of $11.3 \mathrm{~J} / \mathrm{gK}$, which is very close to the liquid-gas phase line. These droplets then are already quite large with sizes of $10^{3}$ atoms and so far there is no evidence for smaller clusters. As the temperature is lowered further the cluster sizes remain in the range $10^{3}-8 \times 10^{3}$ until at an isentrope with $S=8.1 \mathrm{~J} / \mathrm{gK}$, which is nearly coincident with the phase line, very large droplets with $N_{3} \geqslant 10^{4}$ atoms are formed. This behavior can be qualitatively explained by the fact that the smallest stable ${ }^{3} \mathrm{He}$ cluster consists of 29 atoms $^{26}$ so that in order for condensation to be initiated a relatively high atom density is required in the initial phases of the expansion.

\section{Integral cross sections}

The integral cross sections of the droplets are determined from the decrease in the signal at $\theta=0$ (forward peak) with and without scattering gas. Although this small attenuation is not visible in the log plots in Fig. 2, [with the possible exception of Fig. 2(d)] it can be measured with high precision because of the large forward peak count rates. The attenuation is related to the integral cross section $\bar{\sigma}$ of the droplets according to Lambert-Beer's law, ${ }^{33}$

$$
\frac{I}{I_{0}}=\exp \left[-\frac{\bar{\sigma} n_{\mathrm{sec}} L_{\mathrm{eff}} v_{\mathrm{rel}} F_{a 0}}{v_{\mathrm{drop}}}\right],
$$

where $I$ and $I_{0}$ are the intensities of the ${ }^{3} \mathrm{He}$ droplet beam with and without attenuation, $n_{\mathrm{sec}}$ is the number density of the secondary beam gas atoms in the scattering center and $L_{\text {eff }}$ is the effective length of the scattering volume. Moreover, $v_{\text {drop }}$ is the speed of the droplets and $v_{\text {rel }}$ the relative collision velocity. $F_{a 0}$ takes account of the smearing resulting from the velocity distributions of the two nozzle beams ${ }^{36}$ and leads to a correction smaller than about $1 \%$.

The product of the density in the scattering center and the effective length of the scattering volume $\left(n_{\mathrm{sec}} L_{\mathrm{eff}}\right)$ which enters into Eq. (7) was calibrated to within an error of approximately $5 \%$ by measuring the attenuation of a nearly monoenergetic helium atomic beam, for which the integral cross section can be accurately calculated quantum mechanically from the well established interaction potential. ${ }^{37}$ The values of the absolute integral cross sections of the droplets are also listed in the next to last column of Table I.

As discussed in I the measured integral cross sections correspond to the cross sections which would be obtained using a purely classical scattering theory, i.e., without a contribution from forward diffraction. The overall experimental errors are estimated to be about 5\% and result from uncertainties in the absolute determination of the scattering gas density. 


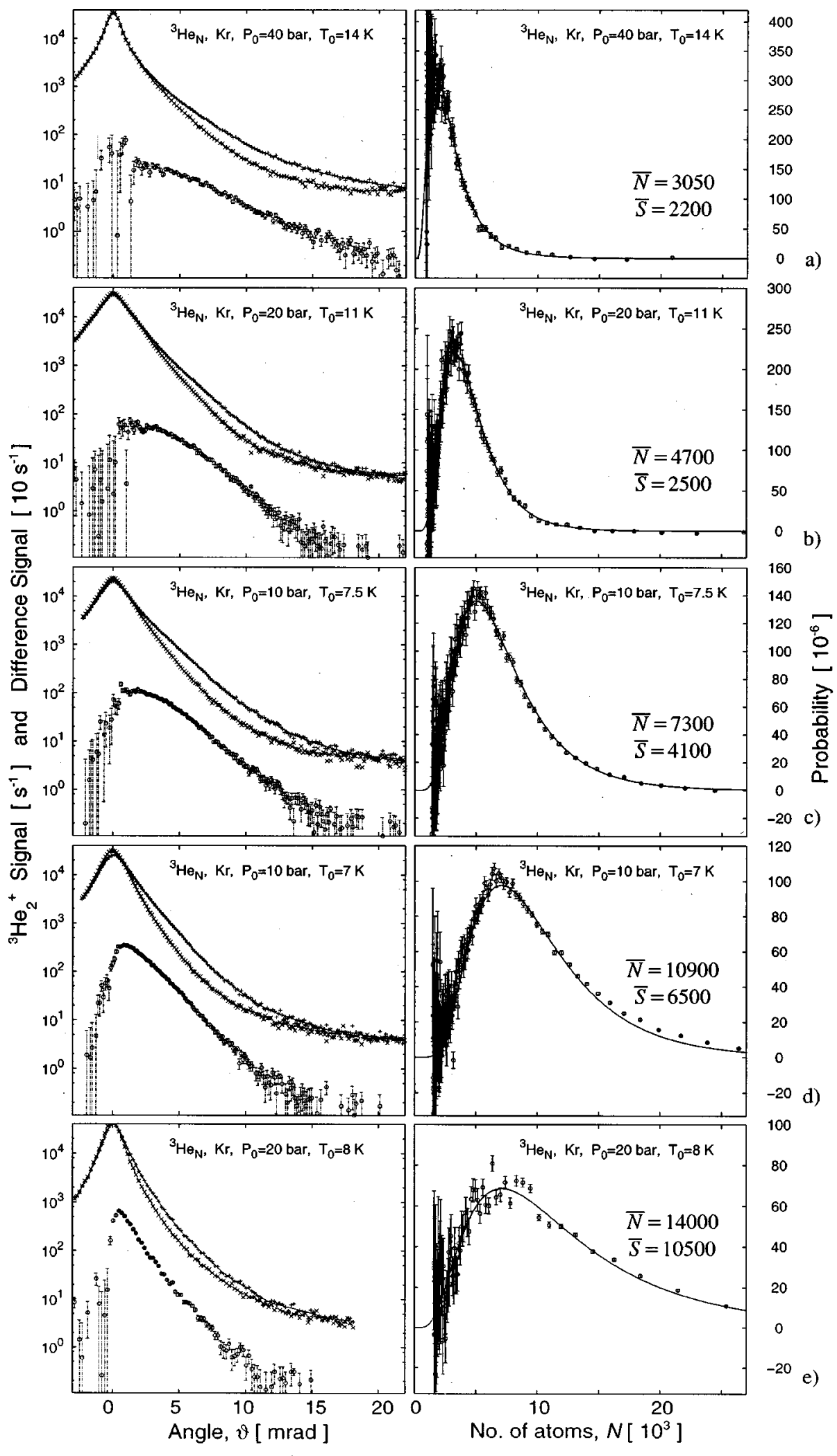

FIG. 2. Five typical measured angular distributions for different source pressures and source temperatures in order of increasing droplet size from top to bottom measured with krypton as secondary beam gas. The measured signals with and without a flag intersecting the secondary beam are plotted on a logarithmic scale as a function of the deflection angle (crosses). Also shown are the weighted differences of the two signals (closed circles) with the standard deviations. In the right column the experimental points are the size probability distributions and the continuous curves are best fit log normal distributions for the following mean number of atoms $\bar{N}$ and the mean standard deviations $\bar{S}$ (a) $\bar{N}=3050, \bar{S}$ =2200; (b) $\bar{N}=4700, \bar{S}=2500$; (c) $\bar{N}=7300, \quad \bar{S}=4100 ;$ (d) $\bar{N}$ $=10900, \quad \bar{S}=6500 ; \quad$ (e) $\bar{N}$ $=14000, \bar{S}=10500$, respectively.

\section{Mean droplet densities}

From the measured cross section $\bar{\sigma}$ an effective mean density $\bar{\rho}$, defined as the density of a uniform sphere with a sharp step edge (liquid drop model) having the same classical integral cross section, is determined:

$\bar{\rho}=\frac{3}{4} \sqrt{\frac{\pi}{\bar{\sigma}^{3}}} \bar{N}$.

The values of $\bar{\rho}$, normalized to the bulk helium density $\rho_{\text {bulk }}=0.01635 \AA^{-3}$, are given in the last column of Table I. 
TABLE I. Experimental results for the mean number of atoms $\bar{N}$ and the half width $\Delta \overline{N_{1 / 2}}$ as a function of source pressure $\left(P_{0}\right)$ and source temperature $\left(T_{0}\right)$, measured with $\mathrm{Kr}$ as a secondary gas. The measured mass distributions from the deflection experiment have been fitted with a log-normal distribution yielding the parameters $\delta$ and $\mu$, see Eq. (4). The mean classical integral cross section $\bar{\sigma}$ is obtained by attenuation of the droplet beam with the secondary beam. The mean density of the droplets $\bar{\rho}$ as a fraction of the known bulk density ( $\rho_{\text {bulk }}=0.01635 \AA^{-3}$ ) is obtained from $\bar{N}$ and $\bar{\sigma}$ using Eq. (8) and is listed in the last column.

\begin{tabular}{rrrrrrrr}
\hline \hline$P_{0}[\mathrm{bar}]$ & $T_{0}[\mathrm{~K}]$ & $\bar{N}$ & $\Delta \overline{N_{1 / 2}}$ & $\delta$ & $\mu$ & $\bar{\sigma}\left[\AA^{2}\right]$ & $\bar{\rho} / \rho_{\text {bulk }}$ \\
\hline 10 & 6.6 & 18783 & 16507 & 0.712 & 9.59 & 14254 & 0.90 \\
10 & 7.0 & 10859 & 9590 & 0.555 & 9.14 & 10188 & 0.86 \\
10 & 7.5 & 7331 & 6371 & 0.522 & 8.76 & 8262 & 0.79 \\
10 & 8.0 & 6778 & 5823 & 0.504 & 8.69 & 7521 & 0.84 \\
10 & 8.5 & 6829 & 5737 & 0.476 & 8.72 & 7835 & 0.80 \\
10 & 8.7 & 8712 & 7757 & 0.581 & 8.90 & 9130 & 0.81 \\
10 & 8.8 & 8932 & 7943 & 0.576 & 8.93 & 9184 & 0.83 \\
20 & 8.0 & 13955 & 12392 & 0.682 & 9.31 & 12425 & 0.82 \\
20 & 8.5 & 7729 & 6735 & 0.526 & 8.81 & 8414 & 0.81 \\
20 & 9.0 & 6443 & 5742 & 0.585 & 8.60 & 7712 & 0.77 \\
20 & 9.1 & 5042 & 4446 & 0.551 & 8.37 & 7003 & 0.70 \\
20 & 9.3 & 4822 & 4157 & 0.509 & 8.35 & 6782 & 0.70 \\
20 & 10.0 & 4316 & 3661 & 0.487 & 8.25 & 5838 & 0.79 \\
20 & 11.0 & 4692 & 4022 & 0.501 & 8.33 & 5902 & 0.84 \\
20 & 11.5 & 4648 & 4073 & 0.537 & 8.30 & 6223 & 0.77 \\
25 & 9.0 & 5052 & 4515 & 0.650 & 8.32 & 6422 & 0.80 \\
25 & 9.5 & 3144 & 2763 & 0.543 & 7.91 & 4830 & 0.76 \\
25 & 9.8 & 2961 & 2633 & 0.575 & 7.83 & 4810 & 0.72 \\
25 & 10.0 & 2545 & 2097 & 0.455 & 7.74 & 4121 & 0.78 \\
25 & 10.5 & 2539 & 2268 & 0.597 & 7.66 & 4056 & 0.80 \\
25 & 11.0 & 2335 & 2090 & 0.617 & 7.57 & 3987 & 0.75 \\
25 & 12.0 & 2463 & 2191 & 0.577 & 7.64 & 3677 & 0.90 \\
25 & 13.0 & 3131 & 2731 & 0.528 & 7.91 & 3844 & 1.07 \\
\hline \hline
\end{tabular}

The experimental data for $\bar{\sigma}$ are plotted versus the measured values for $\bar{N}$ in Fig. 3. For comparison the average integral cross sections expected for uniform liquid drop model spheres with average density $\bar{\rho}$ are plotted for different average relative densities $\bar{\rho} / \rho_{\text {bulk }}$ and are shown as dashed lines. Here the average integral cross section $\bar{\sigma}$ is calculated from the measured log-normal atom number distributions $P(N)$ by means of the following equation:

$$
\overline{\sigma(\bar{N})}=\int_{0}^{\infty} P(N) \pi^{1 / 3}\left(\frac{3 N}{4 \bar{\rho}}\right)^{2 / 3} d N .
$$

As can be seen from Fig. 3 the effective droplet density which comes closest to the experimental points increases from about $0.7 \rho_{\text {bulk }}$, for droplets with $2 \times 10^{3}$ atoms, to about $0.8 \rho_{\text {bulk }}$, for droplets with about $1.5 \times 10^{4}$ atoms. These relative densities are somewhat larger than found for ${ }^{4} \mathrm{He}$ droplets of comparable sizes.

\section{CALCULATED DENSITY PROFILES}

For comparison with these experiments the density profiles of large ${ }^{3} \mathrm{He}$ droplets were calculated using a finite range density functional $\mathcal{E}_{3}(\rho)$ consisting of the ${ }^{3} \mathrm{He}$ part of a more general one described in Ref. 38 and employed there to describe mixed ${ }^{3} \mathrm{He}-{ }^{4} \mathrm{He}$ droplets, whose parameters had been adjusted to reproduce the liquid ${ }^{3} \mathrm{He}$ equation of state and saturation properties, like the energy per atom, saturation (equilibrium) density and incompressibility, as well as the surface tension of the bulk liquid surface at zero temperature and pressure. As in Ref. 35 the large number of ${ }^{3} \mathrm{He}$ atoms in the droplets justifies the use of the extended Thomas-Fermi method to express the kinetic energy density as a function of the particle density and its gradients. ${ }^{18}$

For a given ${ }^{3} \mathrm{He}_{N}$ droplet, the Euler-Langrange equation

$$
\frac{\delta \mathcal{E}_{3}}{\delta \rho}=\frac{\partial \mathcal{E}_{3}}{\partial \rho}-\nabla \frac{\partial \mathcal{E}_{3}}{\partial(\nabla \rho)}+\Delta \frac{\partial \mathcal{E}_{3}}{\partial(\Delta \rho)}=\mu_{3},
$$

where $\mu_{3}$ is the ${ }^{3} \mathrm{He}$ chemical potential, was solved assuming spherical symmetry, using five point formulas to discretize the differential operators and an $R$ step of $\Delta R=0.1 \AA$. Physically acceptable solutions $\rho(R)$ have to go to zero at large distances and be regular at the origin; for a spherically symmetric density this can be achieved by imposing $d \rho / d R=0$ at $R=0$.

As a check on the calculated droplet profiles the EulerLagrange equation was also solved for the bulk liquid surface, in which case the particle density depends only on one Cartesian coordinate $\rho(z)$. For this system, $\rho(-\infty)=\rho_{\text {bulk }}$, 


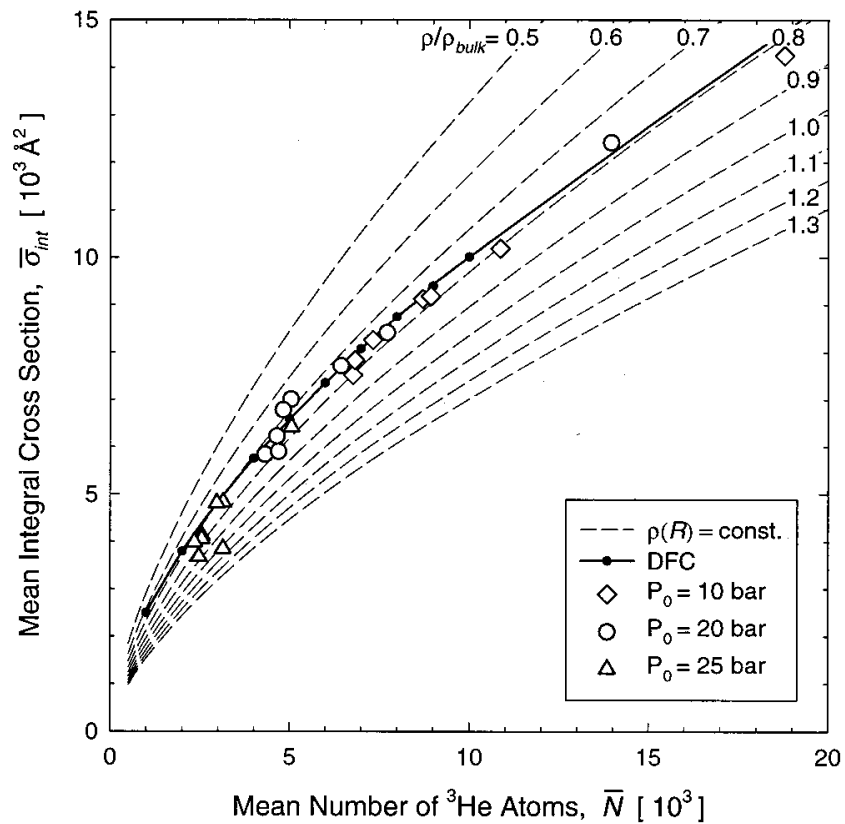

FIG. 3. The measured "classical" integral cross sections (averaged over the measured number distributions) are plotted as a function of the measured mean number of atoms $\bar{N}$. The empty symbols show the experimental results for different source stagnation pressures: $\diamond P_{0}=10$ bars, $\bigcirc P_{0}=20$ bars, and $\triangle P_{0}=25$ bars. The solid line with filled circles is calculated from the DF calculated radial density distributions as described in Sec. IV. For comparison, the classical cross sections of spherical droplets with constant density are indicated as dashed lines for different values of the relative density $\rho / \rho_{\text {bulk }}$ where $\rho_{\text {bulk }}=0.01635 \AA^{-3}$.

$\rho(\infty)=0$, and $\mu_{3}=\mu_{3 \text {,bulk }}$. From the calculated bulk liquid surface profile $\rho_{0}(z)$, the $10-90 \%$ thickness was found to be $t \approx 7.6 \AA$ in excellent agreement with the calculated width of the largest droplets $\left(\bar{N}_{3}=4 \times 10^{4}\right)$

The calculated density profiles are shown in Fig. 4(a). These density profiles appear to be very similar to those reported earlier for ${ }^{4} \mathrm{He}$ droplets. The two major differences are the smaller bulk density of ${ }^{3} \mathrm{He}\left[\rho_{\text {bulk }}\left({ }^{3} \mathrm{He}\right)=1.635\right.$ $\left.\times 10^{-2} \AA^{-3}\right]$ which is $75 \%$ of the ${ }^{4} \mathrm{He}$ density $\left[\rho_{\text {bulk }}\left({ }^{4} \mathrm{He}\right)\right.$ $\left.=2.18 \times 10^{-2} \AA^{-3}\right]$ and the lack of any structure in the falloff region. The radii $R_{0.5}$ calculated for a falloff in density from the bulk value by a factor $\frac{1}{2}$, for both isotopes are, however, very similar and agree with the simple geometrical relationship

$$
\frac{R_{0.5}\left({ }^{4} \mathrm{He}\right)}{R_{0.5}\left({ }^{3} \mathrm{He}\right)}=\left(\frac{\rho_{0}\left({ }^{3} \mathrm{He}\right)}{\rho_{0}\left({ }^{4} \mathrm{He}\right)}\right)^{1 / 3} .
$$

For example, for $N=10^{4}$ atoms for ${ }^{4} \mathrm{He}, \quad R_{0.5}=48 \AA$, whereas for ${ }^{3} \mathrm{He}, R_{0.5}=53 \AA$.

Finally another difference compared to the ${ }^{4} \mathrm{He}$ density profiles appears to be a rather large asymmetry in the density falloff with respect to the $R_{0.5}$ radius. To quantify the asymmetry the radial derivative of the density $\rho^{\prime}(R)$ was calculated for the smallest $\left(N_{3}=10^{3}\right)$ and largest $\left(N_{3}=4 \times 10^{4}\right)$ droplets. Since the results were found to be nearly identical only the radial density falloff and the derivative curve for
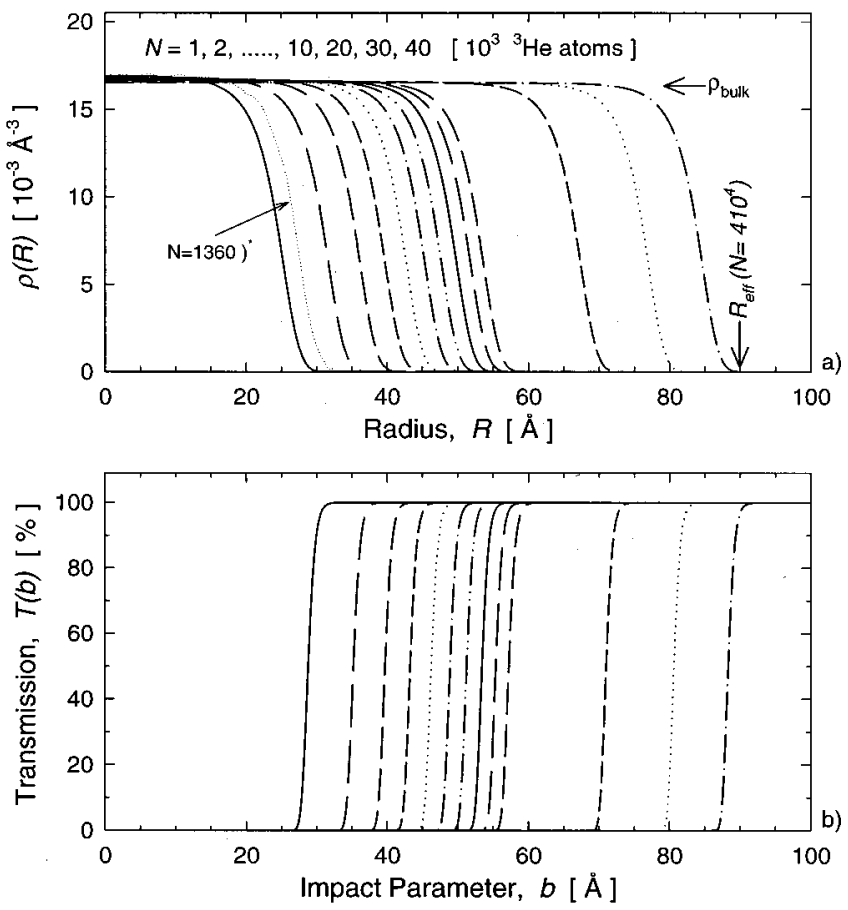

FIG. 4. The density distributions calculated with a densityfunctional method are plotted as a function of the droplet radius $R$. The curves are for droplets with between $N=10^{3}$ and $N=4$ $\times 10^{4}$ atoms in steps of $10^{3}$ up to $N=1 \times 10^{4}$ and in steps of $10^{4}$ up to $4 \times 10^{4}$ atoms. The effective radius $R_{\text {eff }}$ calculated for a droplet with $4 \times 10^{4}$ atoms is shown to illustrate the relation of $R_{\text {eff }}$ to the calculated density distribution. Also shown as a dotted line is the density profile for a ${ }^{3} \mathrm{He}$ droplet with $N_{3}=1360$ (Ref. 23). (b) The corresponding calculated transmission function for a beam of krypton atoms passing through the same droplets shown in (a) are plotted as a function of impact parameter $b$.

$N_{3}=4 \times 10^{4}$ are shown in Fig. 5. The dashed line in the bottom part of Fig. 5 shows the derivative curve if the density falloff were to be symmetric around the radius at which $\rho^{\prime}(R)=0$. Thus the falloff in density from the central region is initially more gradual than in the outermost region.

The DF profiles were also fitted to the analytical function Eq. (1) where the radius $R_{0}$ was fixed so that the droplet has the required number of atoms $N$. The density was taken to be $\rho_{0}=\rho_{\text {bulk }}\left(1+\delta \rho / \rho_{\text {bulk }}\right)$ where $\delta \rho$ takes into account the compression effect caused by the surface tension and the compressibility of liquid ${ }^{3} \mathrm{He}$. This correction has been estimated to be $\mathrm{be}^{18}$

$$
\frac{\delta \rho}{\rho_{\text {bulk }}}=\frac{2}{3} \frac{K \rho_{\text {bulk }} E_{s}}{N^{1 / 3}},
$$

where the surface energy is $E_{s}=8.42 \mathrm{~K}$ and $K \rho_{\text {bulk }}$ $=0.0826 \mathrm{~K}^{-1}$, where $K$ is the compressibility (see Table II and Ref. 29). The correction $\delta \rho / \rho_{\text {bulk }}$ turns out to be $4.6,2$, and $1 \%$ for $N=1000,10000$, and 40000 , respectively. Reasonable fits could be obtained using $\nu=4$ and $a=2.1 \AA$, which yields $t=7.1 \AA$ for the large droplets, in reasonable agreement with the exact DF results. 


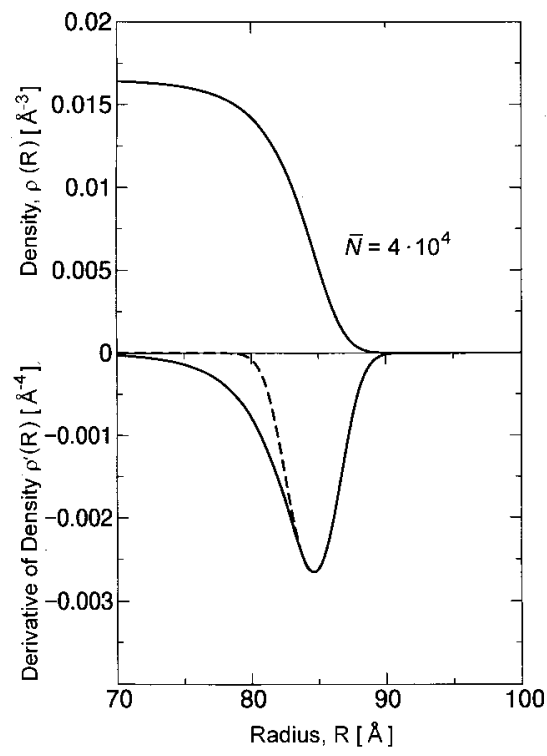

FIG. 5. The calculated outer particle density for a ${ }^{3} \mathrm{He}$ droplet with $N=4 \times 10^{4}$ atoms is plotted as a function of the radius $R$. (b) The solid line shows the radial derivative $\rho^{\prime}(R)$ plotted on the same radius scale. The dashed line shows the $\rho^{\prime}(R)$ curve for the outer region reflected on to the inner region around the point at which $\rho^{\prime}(R)=0$. An identical behavior was found for the density falloff calculated for $N=10^{3}$ atoms.

\section{COMPARISON OF EXPERIMENT AND THEORY}

For comparison with the experiment first the transmission coefficients were calculated as described in I using the following expression:

$$
T(b)=\exp \left(-\int_{\mathbf{s}(b)} \sigma\left(E_{\mathrm{rel}}\right) \rho(\mathbf{z}) d \mathbf{z}\right),
$$

which is based on Beer's law attenuation of particles and the assumption of straight line trajectories for the $\mathrm{Kr}$ atoms passing through the outer regions of the ${ }^{3} \mathrm{He}$ density tails. The method used to calculate the atom-atom scattering cross section $\sigma$ used in Eq. (13) and the justification of the approximation Eq. (13) are given in $\mathbf{I}$. The results for $T(b)$ are presented in Fig. 4(b) where they can be compared with the calculated density profiles. The integral cross sections are then calculated using the "classical" expression

$$
\sigma_{\mathrm{eff}}=2 \pi \int_{0}^{\infty}[1-T(b)] b d b,
$$

which is also justified in I. In a subsequent step the cross sections are averaged over the distribution in the number of atoms $P(N)$ in a similar way as in Eq. (9). The results for the effective radii $R_{\text {eff }}=\sqrt{\sigma_{\text {eff }} / \pi}$, the effective averaged cross section $\sigma_{\text {eff }}$, the average relative densities $\bar{\rho} / \rho_{\text {bulk }}$ and the $10-90 \%$ thickness $t$ obtained from the numerically calculated density profiles are summarized in Table III, where they are compared with the corresponding theoretical values for ${ }^{4} \mathrm{He}$ droplets of the same size reported in I. The calculated average cross sections $\sigma_{\text {eff }}$ and the average relative densities $\bar{\rho} / \rho_{\text {bulk }}$ for the ${ }^{3} \mathrm{He}$ droplets are compared with the experimental values in Fig. 3. The theoretical values, shown as small filled circles, are in excellent agreement with the experimental values which were calculated using Eqs. (8) and (9).

As in I, the surface width was estimated directly from the measured integral cross sections and measured droplet number sizes following the same procedures described above. Equation (1) was used with a fixed value of $\nu=4$ which provided the best fit of the DF calculated profiles and $R_{0}$ and a were fit to the experimental values. The resulting values of $t$ are plotted as a function of $\bar{N}$ in Fig. 6. The mean 10-90\% thickness is found to be about $6.7 \pm 1.3 \AA$, which agrees within the large errors with the DF thicknesses which lie between 7.8 and $7.7 \AA$ for droplets of the same sizes (Table III). A smaller thickness of $5.7 \pm 1.6 \AA$ is obtained if the same symmetric shape function described in I [Eq. (11) which is equivalent to Eq. (1) with $\nu=1$ of this paper] is used. This confirms the asymmetry of the DF calculated falloff region in the case of the ${ }^{3} \mathrm{He}$ droplets shown in Fig. 5 which is not properly accounted for by the symmetric falloff function. It is interesting to note that in the case of the ${ }^{4} \mathrm{He}$ droplets the estimated widths based on the symmetric shape function agreed within the errors with the DF calculations. ${ }^{31}$ This observation provides additional confirmation that the ${ }^{3} \mathrm{He}$ droplets have asymmetric profiles whereas the small asymmetry predicted for ${ }^{4} \mathrm{He}$ droplets could not be experimentally confirmed.

\section{SUMMARY}

By using a combination of scattering techniques (deflection and attenuation), it has been possible to measure the

TABLE II. Comparison of some important physical properties of bulk ${ }^{3} \mathrm{He}$ and ${ }^{4} \mathrm{He}$ and their droplets $\left(N \geqslant 10^{3}\right)$ (Ref. 13).

\begin{tabular}{lccc}
\hline \hline & ${ }^{4} \mathrm{He}$ & ${ }^{3} \mathrm{He}$ & Units \\
\hline Surface tension at 0 K & 0.38 & 0.16 & dyn/cm \\
Chem. potential at 0 K & 7.169 & 2.473 & $\mathrm{~K}$ \\
Bulk particle density at $0 \mathrm{~K}^{\mathrm{a}}$ & $2.18 \times 10^{-2}$ & $1.635 \times 10^{-2}$ & $\AA^{-3}$ \\
Fermi temperature & & 1.75 & $\mathrm{~K}$ \\
Entropy at 2.0 K & 3.85 & 12.95 & $\mathrm{~J} / \mathrm{mole} \mathrm{K}^{3}$ \\
Compressibility at 0 K & 0.120 & 0.361 & $\mathrm{~cm}^{3} / \mathrm{J}$ \\
Temperature of droplets & $0.37( \pm 0.05)$ & $0.15( \pm 0.01)$ & $\mathrm{K}$ \\
\hline \hline
\end{tabular}

${ }^{a}$ At zero pressure.

${ }^{\mathrm{b}}$ References 6 and 7. 
TABLE III. Theoretical results for the effective radii $R_{\text {eff }}=\sqrt{\sigma_{\text {eff }} / \pi}$ and the effective cross sections $\sigma_{\text {eff }}$ obtained using Eq. (14) based on the present DF calculations of density profiles for ${ }^{3} \mathrm{He}$ droplets, are compared with the corresponding values calculated for ${ }^{4} \mathrm{He}$ droplets (Ref. 31). The effective average density $\bar{\rho}$ as a fraction of the known bulk density $\left(\rho_{\text {bulk }}=0.01635 \AA^{-3}\right)$ and the $10-90 \%$ surface thickness $t$ are listed.

\begin{tabular}{ccccccccc}
\hline \hline$N\left[10^{3}\right.$ atoms $]$ & \multicolumn{2}{c}{$\sigma_{\text {eff }}\left[\AA^{2}\right]$} & \multicolumn{2}{c}{$R_{\text {eff }}[\AA]$} & \multicolumn{2}{c}{$\bar{\rho} / \rho_{\text {bulk }}$} & \multicolumn{2}{c}{$t[\AA]$} \\
\cline { 2 - 8 } & ${ }^{3} \mathrm{He}$ & ${ }^{4} \mathrm{He}$ & ${ }^{3} \mathrm{He}$ & ${ }^{4} \mathrm{He}$ & ${ }^{3} \mathrm{He}$ & ${ }^{4} \mathrm{He}$ & ${ }^{3} \mathrm{He}$ & ${ }^{4} \mathrm{He}$ \\
\hline 1 & 2608 & 2083 & 28.81 & 25.75 & 0.61 & 0.64 & 8.0 & 5.6 \\
2 & 3901 & 3135 & 35.24 & 31.59 & 0.67 & 0.69 & 7.9 & 5.6 \\
3 & 4962 & 4000 & 39.74 & 35.69 & 0.70 & 0.72 & 7.8 & 5.7 \\
4 & 5896 & 4763 & 43.32 & 38.94 & 0.72 & 0.74 & 7.8 & 5.7 \\
5 & 6748 & 5459 & 46.35 & 41.69 & 0.73 & 0.76 & 7.8 & 5.7 \\
6 & 7538 & 6106 & 48.99 & 44.09 & 0.75 & 0.77 & 7.8 & 5.7 \\
7 & 8283 & 6713 & 51.35 & 46.22 & 0.76 & 0.78 & 7.7 & 5.7 \\
8 & 8989 & 7292 & 53.49 & 48.18 & 0.76 & 0.78 & 7.7 & 5.7 \\
9 & 9664 & 7843 & 55.46 & 49.97 & 0.77 & 0.79 & 7.7 & 5.7 \\
10 & 10312 & 8373 & 57.29 & 51.63 & 0.78 & 0.80 & 7.7 & 5.7 \\
20 & 15866 & & 71.07 & & 0.81 & & 7.7 & \\
30 & 20469 & & 80.72 & & 0.83 & & 7.6 & \\
40 & 24550 & & 88.40 & & 0.85 & & 7.6 & \\
\hline \hline
\end{tabular}

average densities of large ${ }^{3} \mathrm{He}$ droplets with average numbers of atoms $\bar{N}=2 \times 10^{3}-2 \times 10^{4}$. The results have been compared with density-functional calculations, and overall good agreement has been found. The density functional 10-90\% surface thicknesses vary between $8.0 \AA$ for $N=1 \times 10^{3}$ and $7.6 \AA$ for $N=4 \times 10^{4}$, and are about $30 \%$ larger than those

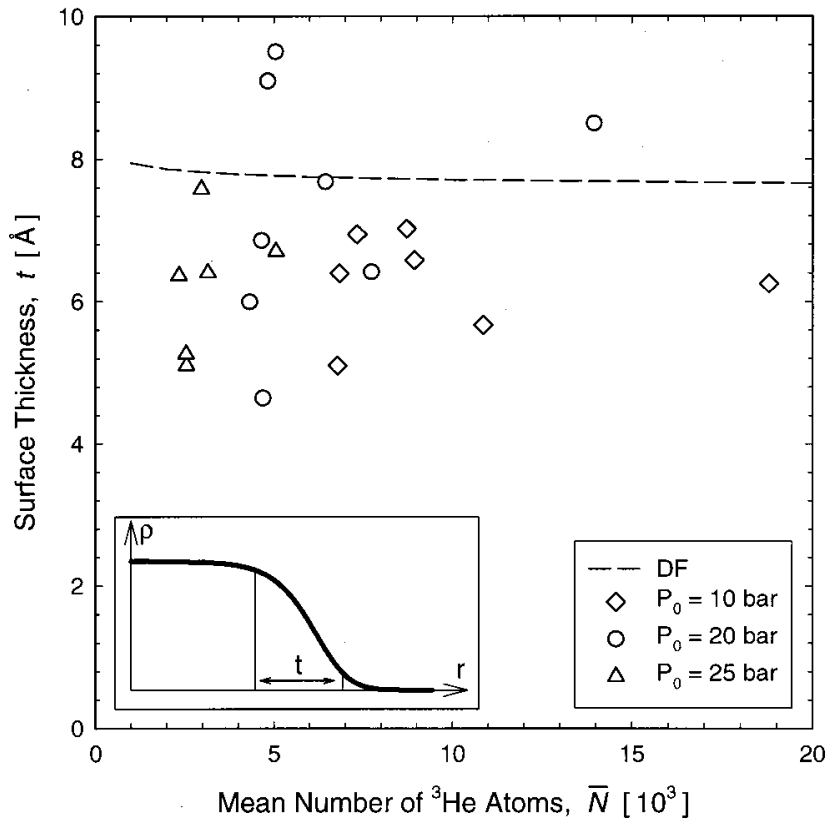

FIG. 6. The experimental $10-90 \%$ surface thickness $t$ of ${ }^{3} \mathrm{He}$ droplets are plotted as a function of the mean number of atoms $\bar{N}$ evaluated from the experimental data assuming an asymmetric density profile [Eq. (1)] with $\nu=4$, as shown in the inset. The mean value of $t$ averaged over all measurements for different sizes is $\bar{t}$ $=6.7 \pm 1.3 \AA$. The present DF values are shown as a dashed line. calculated for ${ }^{4} \mathrm{He}$ droplets of similar sizes (see Table III). Theory and experiments indicate that the density profile of ${ }^{3} \mathrm{He}$ is significantly asymmetric in the falloff region, whereas in our earlier study of ${ }^{4} \mathrm{He}$ droplets no significant evidence for an asymmetry was found.

It is interesting to note that recent $\mathrm{x}$-ray reflectivity measurements of $125-\AA$ thick films of ${ }^{4} \mathrm{He}$ on a silicon substrate, which yield a thickness of $t=6.5 \pm 0.5 \AA,{ }^{39,40}$ are nearly in agreement with the earlier DF calculations which yielded $t$ $=5.7 \AA$ for large droplets. ${ }^{31}$ A fit of the experimental data using a formula similar to Eq. (1) yielded $6.4 \pm 1.3 \AA$. Thus there is now some experimental evidence that large droplets and flat surfaces have very similar thicknesses.

The reasonable agreement found between the densityfunctional calculations and the experiments for both ${ }^{3} \mathrm{He}$ and ${ }^{4} \mathrm{He}$ droplets provides direct confirmation of the calculated density profiles. At the present time there are only a few experiments which are sensitive specifically to the surface region. These include the spectroscopy of alkali ${ }^{41}$ and alkaline-earth $^{42}$ atoms, which are known to be located at the surfaces of ${ }^{4} \mathrm{He}$ droplets and presumably also on ${ }^{3} \mathrm{He}$ droplets. ${ }^{43}$ The metastable excitation by electron bombardment of He droplets has also been shown to occur at the surface of He droplets as well as in the interior and is therefore also indirectly sensitive to the surface density. ${ }^{44}$ In addition, there is now some evidence that the energy position and half width of the observed bands in the photoexcitation spectra of ${ }^{3} \mathrm{He}$ and ${ }^{4} \mathrm{He}$ droplets are correlated with the average density. ${ }^{45}$

\section{ACKNOWLEDGMENTS}

M. Barranco and M. Pi acknowledge support from DGESIC, Spain, Grant No. PB98-1247, and from the Generalitat of Catalunya, Grant No. 2000SGR-00024. We thank F. Dalfovo and J. Boronat for several helpful discussions. 
*Electronic address: jtoenni@gwdg.de

${ }^{1}$ J. P. Toennies and A. F. Vilesov, Annu. Rev. Phys. Chem. 49, 1 (1998).

${ }^{2}$ K. B. Whaley, Int. Rev. Phys. Chem. 13, 41 (1994).

${ }^{3}$ M. Farnik, U. Henne, B. Samelin, and J. P. Toennies, Phys. Rev. Lett. 81, 3892 (1998).

${ }^{4}$ S. Goyal, D. L. Schutt, and G. Scoles, J. Phys. Chem. 97, 2236 (1993).

${ }^{5}$ F. Stienkemeier, J. Higgins, W. E. Ernst, and G. Scoles, Phys. Rev. Lett. 74, 3592 (1995).

${ }^{6}$ M. Hartmann, R. E. Miller, J. P. Toennies, and A. Vilesov, Phys. Rev. Lett. 75, 1566 (1995).

${ }^{7}$ J. Harms, M. Hartmann, J. P. Toennies, A. F. Vilesov, and B. Sartakov, J. Mol. Spectrosc. 185, 204 (1997).

${ }^{8}$ S. Grebenev, J. P. Toennies, and A. F. Vilesov, Science 279, 2083 (1998)

${ }^{9}$ M. Hartmann, R. E. Miller, J. P. Toennies, and A. F. Vilesov, Science 272, 1631 (1996).

${ }^{10}$ P. Sindzingre, M. L. Klein, and D. M. Ceperley, Phys. Rev. Lett. 63, 1601 (1989).

${ }^{11}$ M. V. R. Krishna and K. B. Whaley, J. Chem. Phys. 93, 746 (1990)

${ }^{12}$ J. Harms, M. Hartmann, B. Sartakov, J. P. Toennies, and A. F. Vilesov, J. Chem. Phys. 110, 5124 (1999).

${ }^{13}$ D. Greywall, Phys. Rev. B 27, 2747 (1983).

${ }^{14}$ P. W. Stephens and J. G. King, Phys. Rev. Lett. 51, 1538 (1983).

${ }^{15}$ J. Gspann and H. Vollmar, J. Chem. Phys. 73, 1657 (1980).

${ }^{16}$ S. Stringari, Phys. Lett. 107A, 36 (1985).

${ }^{17}$ V. R. Pandharipande, S. C. Pieper, and R. B. Wiringa, Phys. Rev. B 34, 4571 (1986).

${ }^{18}$ S. Stringari and J. Treiner, J. Chem. Phys. 87, 5021 (1987).

${ }^{19}$ D. M. Brink and S. Stringari, Z. Phys. D: At., Mol. Clusters 15, 257 (1990).

${ }^{20}$ A. Guirao, M. Pi, and M. Barranco, Z. Phys. D: At., Mol. Clusters 21, 185 (1991).

${ }^{21}$ P. Joyes, R. J. Tarento, and J. Van de Walle, Z. Phys. D: At., Mol. Clusters 20, 273 (1991).

${ }^{22}$ L. Serra, F. Garcias, M. Barranco, J. Navarro, and N. Van Giai, Z. Phys. D: At., Mol. Clusters 20, 277 (1991).

${ }^{23}$ S. Weisgerber and P.-G. Reinhard, Z. Phys. D: At., Mol. Clusters 23, 275 (1992).

${ }^{24}$ C. Yannouleas and U. Landman, J. Chem. Phys. 105, 8734
(1996).

${ }^{25}$ C. Yannouleas and U. Landmann, Phys. Rev. B 54, 7690 (1996).

${ }^{26}$ M. Barranco, J. Navarro, and A. Poves, Phys. Rev. Lett. 78, 4729 (1997).

${ }^{27}$ R. Guardiola and J. Navarro, Phys. Rev. Lett. 84, 1144 (2000).

${ }^{28}$ M. Barranco, D. M. Jezek, E. S. Hernandez, J. Navarro, and L. Serra, Z. Phys. D: At., Mol. Clusters 23, 257 (1993).

${ }^{29}$ S. Stringari and J. Treiner, Phys. Rev. B 36, 8369 (1987).

${ }^{30}$ M. Barranco, M. Pi, A. Polls, and X. Vin̄as, J. Low Temp. Phys. 80, 77 (1990).

${ }^{31}$ F. Dalfovo, J. Harms, and J. P. Toennies, Phys. Rev. B 58, 3341 (1998).

${ }^{32}$ M. Lewerenz, B. Schilling, and J. P. Toennies, Chem. Phys. Lett. 206, 381 (1993).

${ }^{33}$ M. Lewerenz, B. Schilling, and J. P. Toennies, J. Chem. Phys. 102, 8191 (1995).

${ }^{34}$ J. Harms, J. P. Toennies, and E. L. Knuth, J. Chem. Phys. 106, 3348 (1997).

${ }^{35}$ M. Pi, R. Mayol, and M. Barranco, Phys. Rev. Lett. 82, 3093 (1999).

${ }^{36}$ K. Berkling, R. Helbing, K. Kramer, H. Pauly, C. Schlier, and P. Toschek, Z. Phys. 166, 406 (1962).

${ }^{37}$ K. T. Tang and J. P. Toennies, Z. Phys. D: At., Mol. Clusters 1, 91 (1986).

${ }^{38}$ M. Barranco, M. Pi, S. M. Gatica, E. S. Hernandez, and J. Navarro, Phys. Rev. B 56, 8997 (1997).

${ }^{39}$ R. M. Townsend, J. Gryko, and J. Rice, J. Chem. Phys. 82, 4391 (1985).

${ }^{40}$ K. Penanen, M. Fukuto, R. K. Heilmann, I. F. Silvera, and P. S. Pershan, Phys. Rev. B 62, 9621 (2000).

${ }^{41}$ F. Stienkemeier, J. Higgins, W. E. Ernst, and G. Scoles, Phys. Rev. Lett. 74, 3592 (1995).

${ }^{42}$ F. Stienkemeier, F. Meier, and H. O. Lutz, Eur. Phys. J. D 9, 313 (1999).

${ }^{43} \mathrm{We}$ are not aware of any experiments involving alkali atoms on ${ }^{3} \mathrm{He}$ droplets. DF calculations similar to those described here indicate, however, that alkali atoms are located at the surface of ${ }^{3} \mathrm{He}$ droplets [F. Garcias and M. Barranco (unpublished)].

${ }^{44}$ U. Henne and J. P. Toennies, J. Chem. Phys. 108, 9327 (1998).

${ }^{45}$ K. von Haeften, A. R. B. de Castro, M. Joppien, L. Moussavizadeh, R. von Pietrowski, and T. Möller, Phys. Rev. Lett. 78, 4371 (1997). 